\title{
Increasing the quantum efficiency of InAs/GaAs QD arrays for solar cells grown by MOVPE without using strain-balance technology
}

Nikolay A. Kalyuzhnyy , Sergey A. Mintairov, Roman A. Salii , Alexey M. Nadtochiy , Alexey S. Payusov , Pavel N. Brunkov , Vladimir N. Nevedomsky, Maxim Z. Shvarts , Antonio Martí , Viacheslav M. Andreev and Antonio Luque

\begin{abstract}
Research into the formation of InAs quantum dots (QDs) in GaAs using the metalorganic vapor phase epitaxy technique is presented. This technique is deemed to be cheaper than the more often used and studied molecular beam epitaxy. The best conditions for obtaining a high photoluminescence response, indicating a good material quality, have been found among a wide range of possibilities. Solar cells with an excellent quantum efficiency have been obtained, with a sub-bandgap photo-response of $0.07 \mathrm{~mA} / \mathrm{cm}^{2}$ per QD layer, the highest achieved so far with the InAs/GaAs system, proving the potential of this technology to be able to increase the efficiency of lattice-matched multi-junction solar cells and contributing to a better understanding of QD technology toward the achievement of practical intermediate-band solar cells.
\end{abstract}

KEYWORDS

MOVPE; solar cells; quantum dots; InAs/GaAs; $Q D$ array; photoluminescence; photocurrent

\section{INTRODUCTION}

A great deal of interest came about at the beginning of this new century in the so-called third-generation solar cells (SCs) [1]. One of these concepts was the intermediate-band (IB) SC [2], which promised to grow the equivalent of a triple-junction $\mathrm{SC}$ in a single-junction SC. This is achieved by creating bandgap-energy electron-hole pairs using two subbandgap photons that use an energy band (or set of levels) located within the semiconductor's energy bandgap as a stepping stone for the electron-hole generation process. This method allows photons of energy lower than the bandgap to be made use of. An alternative concept was associated with the possibility of producing more than one electron-hole pair with photons of at least twice the energy bandgap [3]. Both concepts may be materialized with quantum dots (QDs)
$[3,4]$, and they are related by fundamental considerations [5]. Considerable scientific progress has been made in both concepts [6,7], but they are still in a research phase prior to their possible practical application.

The confined states around the QD have energies located within the bandgap of the host semiconductor which can be used as an IB. The first IB SC prototype [8] was made using InAs QDs grown by molecular beam epitaxy (MBE) in GaAs (refer to Ramiro et al. [9] for more references). The highest efficiency achieved by MBE was $18.32 \%$ in 2009 [10], but in all cases, the increase in current brought about by the QDs, as compared with a control cell made with the same structure and process while excluding the QD formation, is very small and has been accompanied by a reduction in the voltage with an overall reduction in efficiency. IB SCs have also been made with other QD/host semiconductors [11,12]. Most 
of these experiments have been carried out using MBE. Technology to form self-organized QDs was researched intensively within the framework of the MBE technique, which ensures ample feasibilities for controlling the properties of the QD arrays [13]. For devices grown using the MBE technique, QDs have already proven to be practical. In particular, lasers based on QDs have demonstrated extremely low threshold current densities and weak temperature sensitivity [14,15]. Suppression of the lateral charge carrier transport in a QD array has allowed micro-resonators of super-small dimensions based on QDs [16] to be created. Multilayer QD arrays have allowed a super-wide spectrum of laser generation demanded for applications in multichannel optic data transmission [17] to be achieved.

However, because of its slow growth rate, MBE is considered an expensive technique. In photovoltaics, where cost is a major constraint, the use of the faster metalorganic vapor phase epitaxy (MOVPE) is more attractive. In fact, it is the technique used for the commercial production of high-performance, III-V multi-junction SCs (an efficiency of about 40\%) either based on the lattice-matched (to the Ge substrate) $\mathrm{Ga}_{0.51} \mathrm{In}_{0.49} \mathrm{P} / \mathrm{Ga}_{0.99} \mathrm{In}_{0.01} \mathrm{As} / \mathrm{Ge}$ heterostructure or the metamorphic, (lattice-mismatched) $\quad \mathrm{Ga}_{\mathrm{x}} \mathrm{In}_{1-{ }_{\mathrm{x}} \mathrm{P} /}$ $\mathrm{Ga}_{\mathrm{y}} \mathrm{In}_{1-\mathrm{y}} \mathrm{As} / \mathrm{Ge} \mathrm{SCs}$ intended to produce a better spectral matching at the expense of the material quality. Some champion SCs are, however, made using the MBE technique $[18,19]$, but they might not go on to a commercial stage because of their complexity.

Quantum dot SCs, grown by MOVPE, were demonstrated by [20] using five layers of QDs with GaP strain balance layers between the QD layers. A reasonable current increase was observed with respect to a GaAs control cell (without QDs), but a degradation of the open-circuit voltage was significant. In [21,22], an enhanced photocurrent was achieved without any degradation of the open-circuit voltage, with arrays of five layers of QDs grown with thin $(11 \mathrm{~nm})$ spacer layers; by applying an optimized antireflection coating, QD SCs with an efficiency of $18.7 \%$ (AM1.5G, 1 sun) were achieved. However, the authors found that the presence of carrier recombination at the QDs attributed to interfacial defects even for five layers of QDs.

To suppress defect formation when the number of layers of QDs is increased, we can either thicken the spacers between the QD layers or use strain-compensation layers. The latter approach (strain-compensation layers) was successfully demonstrated in [23], where GaAs SC prototypes, made by MOVPE using InAs QDs, were presented, with a higher efficiency $(14.3 \%)$ than a control cell manufactured using the same process, but without QDs. This was, to our knowledge, the first time this higher (than control cell) efficiency was achieved with QD SCs made with III-V materials. The main reason for this is the high structural perfection of the SCs as a result of the use of strain balance GaP layers and the achievement of a rather high sub-bandgap photon absorption by the QDs. P can be introduced with the MBE technique, but its pyrophoric properties make most MBE equipment owners reluctant to use it. On the other hand, it is standard in MOVPE (where it is introduced as $\mathrm{PH}_{3}$ ).

This paper looks at the research and development of a process for forming InAs QDs in GaAs using MOVPE and excluding any strain balance material, with the purpose of obtaining high-quality SC structures and a strong photon absorption by the QDs. This exclusion is to avoid the change of the barrier material for the QDs, which if it has a smaller bandgap than the GaAs, it may induce additional current that may be erroneously interpreted as sub-bandgap current. This is the case in the MBE experiments of reference [24] (although in this case, the authors have correctly interpreted the enhancement in current).

To achieve this aim, extensive research into the growth conditions in structures like SCs with a single-layer of QDs has been carried out, and once the best conditions have been identified, several layers of QDs have been formed, with the QD layers separated by spacers think enough $(40 \mathrm{~nm})$ as to suppress the QDs' interfacial defect formation. A diode with the structure of the SC but without the top metal grid has been prepared to measure the quantum efficiency (QE). In total, more than 100 runs have been made, of which the most relevant are discussed here.

We point out that the results obtained in this paper are of interest in current lattice-matched triple junction SCs. In these cells, the efficiency is limited by the middle cell photocurrent, if the lattice constant is to be matched to that of the Ge. The introduction of the QDs does not affect the middle cell lattice constant but extends its spectral sensitivity by up to $1.3 \mu \mathrm{m}$, thus increasing its current. Although quantum wells have been proposed for this purpose, the inherent strain compensation of the QD structure, made evident by the high integrated photoluminescence (PL) obtained with the QDs, may be an advantage in favor of the latter nanostructure.

According to our results, we are confident that we have achieved the highest increase in photocurrent per QD layer measured for SCs with GaAs barrier material to date.

The paper is organized classically. The experiments and characterization techniques are first described, followed by a discussion of the results. Finally, some conclusions are drawn

\section{EXPERIMENTAL PROCEDURE}

All experimental and device structures have been obtained using the MOVPE technique on an R\&D installation with a horizontal reactor at low pressure (100 mbar). Metal-organic compounds were used as sources of Group III elements (trimethylgallium, trimethylaluminum, and trimethylindium). The concentration of trimethylindium molecules in hydrogen at the output of the thermostat was kept constant by means of an Epison 4 controller to exclude the effect of the unstable trimethylindium vapor 
pressure on the composition of the solid solution with In. Arsine $\left(\mathrm{AsH}_{3}\right)$ was used as a source of the Group V element (As). Experiments were carried out on GaAs substrates, with the precise orientation of the surface (100) and on substrates misoriented to the (111) direction by 2 and $6^{\circ}$.

In reference [25], the MOVPE growth characteristics in the InAs-GaAs system were researched with the help of experimental $\mathrm{In}_{\mathrm{x}} \mathrm{Ga}_{1-{ }_{x}} \mathrm{As} / \mathrm{GaAs}$ quantum wells. Dependencies of the In concentration in the $\mathrm{In}_{\mathrm{x}} \mathrm{Ga}_{1-{ }_{\mathrm{x}}} \mathrm{As}$ solid solution were determined. Based on the analysis of the PL spectra, the dependencies of the InAs growth rate on the In mole flow (As is always in excess) in a wide temperature range $\left(480-700^{\circ} \mathrm{C}\right)$ were calculated. The epitaxial dependencies obtained for the InAs-GaAs system were used in creating heterostructures with QDs.

To investigate the InAs QD parameters, two types of heterostructures were grown: a-heterostructures with InAs QDs embedded in a GaAs matrix for transmission electron microscopy (TEM) and PL observation and b-heterostructures with open single QD layers for atomic force microscopy (AFM) observation.

As shown in Figure 1, the a-heterostructures include wide bandgap $\mathrm{Al}_{0.3} \mathrm{Ga}_{0.7} \mathrm{As}$ barriers at the bottom and the top, which protect the active region of the InAs QD layer and GaAs matrix against electron-hole recombination. The total thickness of the GaAs was $500 \mathrm{~nm}$. However, the growth parameters of the GaAs grown directly after QDs ("caplayer") were different from those of the rest of the structure. That is, the growth temperature for the QD and GaAs "caplayer" varied within the $520550^{\circ} \mathrm{C}$ range, whereas the remaining layers of the experimental heterostructures were grown at the fixed temperature of $700^{\circ} \mathrm{C}$. It is important that the GaAs "underlayer" be grown in the mass-transport regime [26], because the perfect quality of the GaAs surface is necessary for the deposition of the QDs [25].
The b-heterostructures, with open single QD layers, were grown in a similar way with the only difference that QDs were not covered by GaAs after the InAs deposition and QD formation. Subsequently, they cooled down and were taken out of the MOVPE reactor.

A technological characteristic when growing all of the heterostructures was that a constant, fairly high growth rate of not less than $1.5 \mu / \mathrm{h}$ was chosen for the bulk GaAs binary component, except in the vicinity of the QDs. This is because SCs, with or without QDs, have to be grown quickly for reasons of cost.

A study of the surface topography of samples with the open b-heterostructure with a single QD layer was carried out using the AFM method on a Dimension 3100 (Veeco) microscope at room temperature and an atmospheric humidity of $\sim 20 \%$ using AFM probes with the tip curvature radius below $10 \mathrm{~nm}$.

The cross section of the a-heterostructures was examined by TEM. The samples were prepared for the TEM study using the conventional procedure of preliminary mechanical thinning, followed by final ion beam milling $(3.5 \mathrm{keV} \mathrm{Ar}+$ ions). The TEM study was carried out with a JEOL JEM $2100 \mathrm{~F}$ and a JEOL-2000FX II microscopes at 200 and $300 \mathrm{kV}$ accelerating voltage at the Ioffe Institute of $\mathrm{St}$ Petersburg, Russia, and at the Electron Microscopy service of the University of Zaragoza (UZ), Spain (at the behest of and in collaboration of the Polytechnic University of Madrid, Spain), respectively.

To obtain the PL spectra of the a-heterostructures, a $532 \mathrm{~nm} \mathrm{Nd}$ : YAG laser of $350 \mathrm{~mW}$ power was used as a source of radiation. Radiation was focused on an МДР-23 monochromator input slit through a collecting lens. To study the temperature dependencies of the PL spectra, samples were placed into a closed-cycle liquid helium cryostat CTI-CRYOGENICS, with a possibility of maintaining the temperature within the $10-300 \mathrm{~K}$ range.

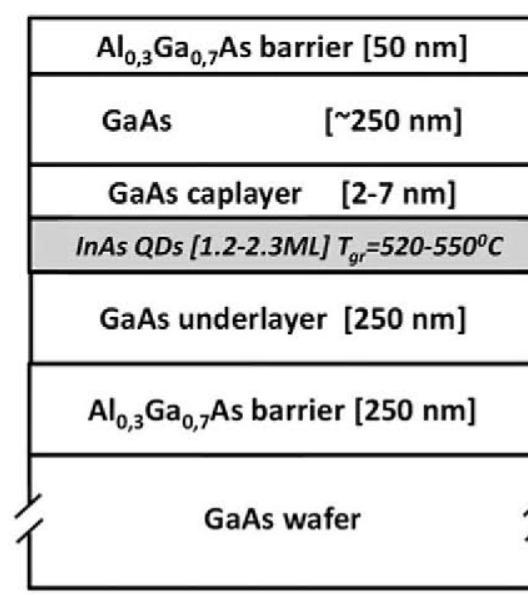

(a)

\begin{tabular}{|c|}
\hline $\operatorname{lnAs} Q D S[1.2-2.3 M L] T_{g r}=520-550^{\circ} \mathrm{C}$ \\
\hline GaAs underlayer $[250 \mathrm{~nm}]$ \\
\hline $\mathrm{Al}_{0,3} \mathrm{Ga}_{0,7}$ As barrier $[250 \mathrm{~nm}]$ \\
GaAs wafer
\end{tabular}

(b)

Figure 1. a- and b-heterostructures (Figures [a] and [b], respectively) for atomic force microscopy (AFM), transmission electron microscopy (TEM), and photoluminescence (PL) characterization. The b-heterostructures are only used for AFM characterization. 
All measurements were taken by means of a cooled $\mathrm{Ge}$ optical radiation detector using a standard synchronous detection procedure.

To find the maximum PL intensity of the aheterostructures, the amount of InAs deposited was varied at different epitaxial and structural parameters: the QD growth rate (varied within $0.0625-0.25$ monolayers per seconds $[\mathrm{ML}] / \mathrm{s}$ ), the thickness (varied within $5-50 \mathrm{~nm}$ ), and the GaAs caplayer growth rate (varied within 2 $4 \mathrm{~A} / \mathrm{s}$ ). A number of other epitaxial parameters (e.g., a ratio of Group V/III atoms in the gas phase together with an optimum range of temperatures of InAs QD deposition), at which the QDs were deposited, have been chosen according to the research carried out earlier on the growth characteristics of the InAs-GaAs system [25].

As a result of the optimization of the PL intensity, the growth conditions of QD arrays have been established. QD arrays were embedded into the active area of the GaAs $\mathrm{SC}$ structure. The GaAs SC structures were based on a GaAs p-i-n diode having 10-15 InAs QD layers in the i region with a $3 \mu \mathrm{m}$-thick base, $0.5 \mu \mathrm{m}$-thick emitter, and $\mathrm{Al}_{0.8} \mathrm{Ga}_{0.2} \mathrm{As}$ and $\mathrm{Al}_{0.3} \mathrm{Ga}_{0.7} \mathrm{As}$ layers for the window and the back surface field layers, respectively. For a comparative analysis of the characteristics, reference GaAs $\mathrm{SC}$ structures were also grown without QDs in the active region.

Based on the GaAs SC structures grown, control photodiodes (SCs without a metal grid on the top) were manufactured, and their spectral photocurrent in the $320-1080 \mathrm{~nm}$ range was measured.

The multifunctional installation at the Ioffe Institute has been used for measuring the external quantum efficiency (EQE) and the reflectance of the SCs. The measuring hardware included an unblocked ultra-violet halogen light source, a grating monochromator with $2 \mathrm{~nm} / \mathrm{mm}$ dispersion within 300-1200 $\mathrm{nm}$ wavelength scanning range, and an optical chopper of $90 \mathrm{~Hz}$ and high sensitivity lock-in electronics. The EQE measurement was controlled by a laptop. The lock-in technique allows weak output signals at the QD sensitivity range to be detected confidently.

\section{RESULTS AND DISCUSSION}

One of the basic parameters affecting crystalline perfection, QD density, and its size is the condition of the GaAs surface on which nucleation of the InAs occurs [27]. To estimate the planarity of the GaAs surface and its effect on the formation of InAs QDs, research into bheterostructures with open single QD layers grown on GaAs substrates with the (100) surface misorientation of 0,2 , and $6^{\circ}$ (hereafter 0,2 , and $6^{\circ}$ substrates, respectively) has been carried out

Analysis of the AFM images of heterostructures with InAs deposited on 0 and $2^{\circ}$ substrates has allowed the quality of the GaAs underlayer to be established. This presents a wavy surface, and the InAs islands are deposited in the lower part of the waves (Figure 2[a] and [b]). This surface roughness is apparently associated with a threedimensional growth mode, which arises if the rate of nucleation island growth on the surface (the lateral component of the growth rate) appears to be lower than the vertical component of the growth rate [26]. In this case, the nucleation islands are not confined into a planar epitaxial layer, and as a result, faceted pyramids arise. This is clearly seen in the $0^{\circ}$ substrate (Figure 2[a]). The change in the surface energy in the lower part of the wavy surface formed during three-dimensional growth led to the InAs island formation being energetically advantageous in these regions. One can improve the planarity of the 0 and $2^{\circ}$ substrates through a substantial reduction in the growth rate by growing the GaAs underlayer at a low temperature, but as said before, this is undesirable from the point-of-view of cost.

The study of the AFM images of the structures grown on the $6^{\circ}$ substrates has demonstrated an improvement in the GaAs underlayer surface planarity (Figure 2[c] and [d]). This is valid for QDs deposited on the GaAs underlayer at both 550 and $520^{\circ} \mathrm{C}$. The GaAs underlayer planarity indicates a rise in the lateral component of the epitaxial layer growth for the $6^{\circ}$ substrates. This is explained by the increase in the number of steps on the substrate's vicinal surface, which presents more energetically advantageous places near these steps for nucleating the planar layer.

Atomic force microscopy images allow the quality of GaAs surface obtained at the described growth conditions to be estimated, but they are quite useless from the viewpoint of the statistical analysis of the density and size of the objects. The problem of AFM studies is that the conditions for forming InAs islands without capping (b-heterostructure, Figure 1) differ from those in which InAs QDs are capped in a real device (like in an a-heterostructure, Figure 1). In the first case, after nucleation, the InAs islands are exposed on the hot surface for a long period, and their density and size evolve very rapidly during cooling under the arsine flow. Therefore, the resulting density and size of these objects after such slow quenching are completely different from the result obtained from the InAs QDs after fast capping when the QDs are completely frozen.

Indeed, the TEM image (Figure 3[a]) of capped a-heterostructure shows that the density of QDs is at least an order of magnitude larger than in the AFM images. The lateral QD sizes are completely different too in comparison with the InAs islands observed with AFM. In Figure 3(b), a single QD of about $4 \times 12 \mathrm{~nm}$ can be observed, much smaller than the size of the big islands in the AFM picture. Moreover, the TEM image shows a more homogeneous QD size distribution, while the AFM images show an extremely inhomogeneous size distribution of the InAs islands. Furthermore, a rather thick wetting later is also visible along the picture on Figure 3(a).

A-Heterostructures grown on substrates with different misorientation were investigated by PL. The PL intensity from the samples grown on 0 and $2^{\circ}$ was too low, most 

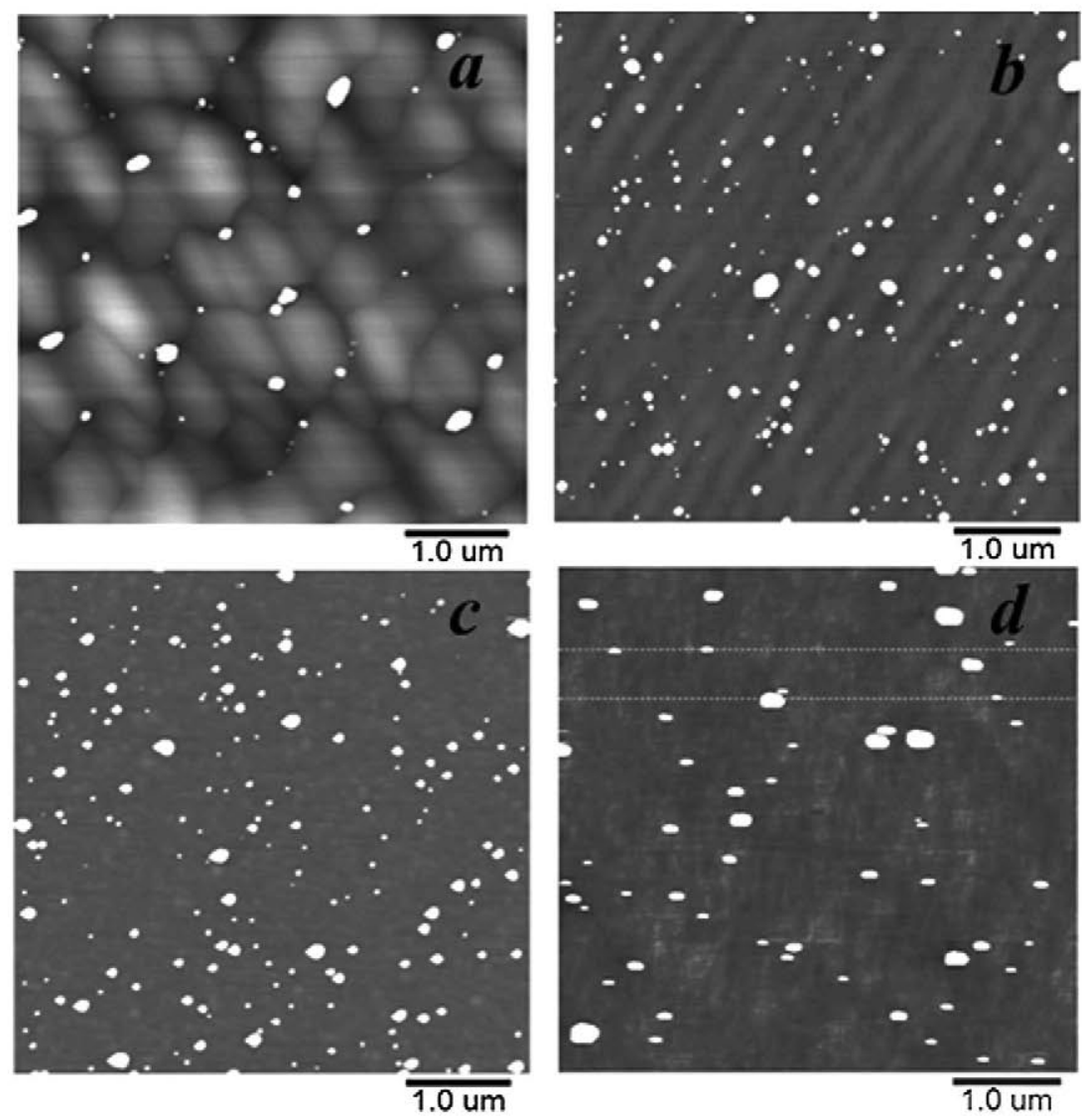

Figure 2. AFM images of InAs islands with a thickness of 1.7 monolayer $(\mathrm{ML})$ deposited on a $\mathrm{GaAs}(100)$ surface with misorientation: (a) 0 , (b) 2 , and (c) $6^{\circ}$ at an $\ln A$ s growth temperature of $550^{\circ} \mathrm{C}$ and (d) $6^{\circ}$ at an $\ln A$ s growth temperature of $520^{\circ} \mathrm{C}$.

probably because of defects which appeared in QDs as a result of the growth on a "wavy" surface (Figure 2[a] and [b]). The structures grown on $6^{\circ}$ at $520^{\circ} \mathrm{C}$ (Figure 2[d]) demonstrated the best PL intensity in comparison with the other samples.

The integrated PL measured on samples grown at temperature of $520^{\circ} \mathrm{C}$ for the undergrown layer and $6^{\circ}$ of surface misalignment is presented in Figure 4. It represents a typical case of a Stranski Krastanov [28] mode of growth. When the QD material is deposited, continuous monolayers of InAs are first formed. The PL signature in this situation, corresponding to a quantum well, is narrow

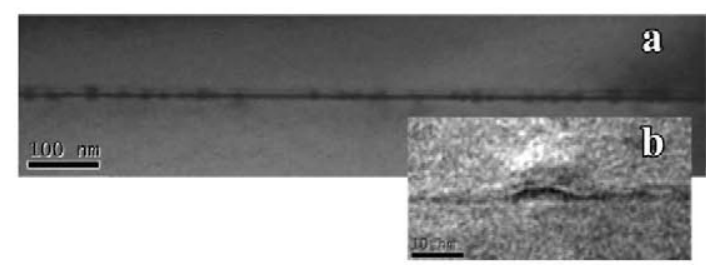

Figure 3. TEM image of cross section of a sample with quantum dots (QDs) covered with a 5 nm-thick GaAs caplayer obtained at loffe: (a) layer with InAs QDs at lower magnification and (b) one separate $\ln A s Q D$. and high, but the integrated PL is weak. The GaAs has a lattice constant of $5.63 \AA$, whereas that of the InAs is 6.05. Because of the difference in lattice constant between

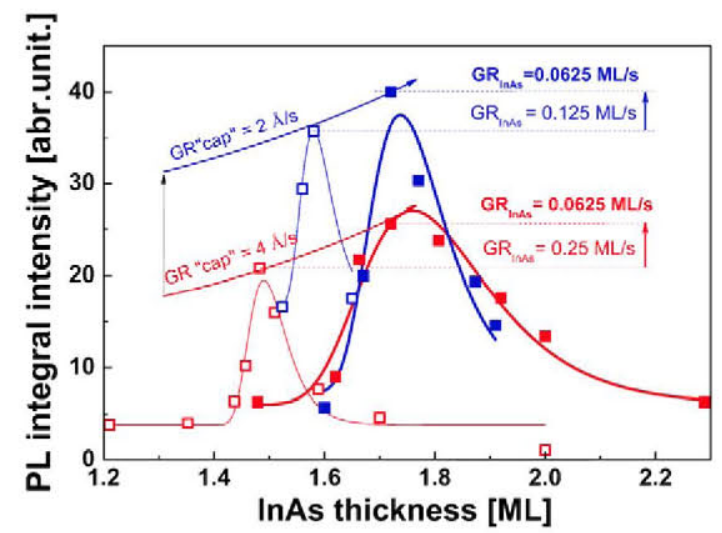

Figure 4. Dependences of the integrated PL intensity on InAs thickness grown at different $\ln A s$ growth rates and GaAs caplayers. Open squares for $0.25 \mathrm{ML} / \mathrm{s}$; solid squares 0.0625 $\mathrm{ML} / \mathrm{s}$. Red color for cap layer growth of $4 \hat{A} / \mathrm{s}$; blue color for

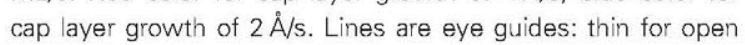
squares and thick for solid squares. 
the InAs and the GaAs, the former is grown under heavy compression conditions. When a certain InAs thickness called the critical thickness, measured in ML, has been reached, the surface breaks, and a tridimensional structure with InAs islands is formed. The PL changes dramatically, giving rise to a broader signature, whose integrated value is substantially larger. If we continue the deposition of InAs layers, the QDs grow bigger, and an optimal thickness is found, in which the PL is highest. This is deemed to be the optimal situation. If we continue depositing InAs monolayers, the QDs grow further, but the integrated PL becomes lower. The conditions with maximum PL are those selected for making SCs.

For QDs deposited at a rather high rate $\left(\mathrm{GR}_{\mathrm{InAs}}=0.25\right.$ $\mathrm{ML} / \mathrm{s}$ ), about $1.5 \mathrm{ML}$ of the InAs corresponds to the optimal thickness with maximum PL intensity (Figure 4, red bell-shaped line with open squares). By decreasing the QD growth rate to $\mathrm{GR}_{\mathrm{InAs}}=0.0625 \mathrm{ML} / \mathrm{s}$, the maximum PL intensity is shifted to $1.7 \mathrm{ML}$ (Figure 4, red bell-shaped line with solid squares), and the range of InAs thickness, in which defect-free QDs with good PL are formed, becomes wider. We believe that these changes are associated with the intermixing of Ga and In atoms in the sublattice of Group III of QD material, which leads to an increase in the lattice constant, and consequently, to an increase in the deposited layer's critical thickness (from 1.43 to $1.60 \mathrm{ML}$ ). Actually, InAs has a quite narrow technological window for forming QDs on GaAs as a result of the large lattice constant difference. But if the InAs QD growth rate is slow, Ga begins to become inserted into the group III sublattice, and InGaAs QDs are formed, thus increasing the critical thickness and widening the ranges in which QDs with good PL are formed; this is the result of reducing the difference between the lattice constants of the QDs and GaAs.

Furthermore, by reducing the InAs growth rate, the maximum PL intensity is also increased by $\sim 12 \%$ (red drawings).

The blue symbols appear when the growth rate of the caplayer is decreased from 4 to $2 \AA / s$. Qualitatively, the behavior is the same. For the faster rate of growth of the InAs layers $(0.25 \mathrm{ML} / \mathrm{s})$, the optimal thickness is 1.55 ML; for the lower growth rate $(0.0625 \mathrm{ML} / \mathrm{s})$, the optimal thickness is $1.7 \mathrm{ML}$. However, the integrated PL intensity is much increased for the slow grown caplayers $(2 \AA / s)$, up to $20 \%$ more. Furthermore, by decreasing the caplayer growth rate from 4 to $2 \AA / s$, the PL intensity rises substantially (blue symbols). The relative increase in the PL intensity by reducing the caplayer growth rate is much greater than by reducing the growth rate of the QDs. PL intensity increased by $\sim 60 \%$ by reducing caplayer growth rate by half, independently of the InAs growth rate.

Note that the half-width of the bell-shaped curves is somewhat modified by decreasing the caplayer growth rate, but the effect is not as critical as it is in the case of changing the rate of growth of InAs. As mentioned in the preceding texts, we believe that good QD formation is associated with increasing Ga concentration in QDs by widening the range of InAs, as a result of $\mathrm{Ga}-\mathrm{In}$ intermixing. Caplayer growth rate effects on the $\mathrm{Ga}-\mathrm{In}$ intermixing are weaker, because the GaAs caplayer growth rate is much higher than the InAs deposition rate.

The thickness of the caplayer within 5-7 nm affects the integrated PL intensity at room temperature weakly (Figure 5). Two PL lines (QD1 and QD2), which are seen in Figure 5, might be indicative of a bimodal distribution of the QD array. An increase in the thickness of the "caplayer", in this case, results in an increase of both the wavelength (red shift) of the (QD2) peak and its relative intensity by $30 \%$. The red shift of the QD2 suggests larger QDs. This observation speaks in favor of the assumption that larger thickness of the GaAs caplayer covers, the higher QDs, because it prevents them from dissolving.

The integrated PL intensity versus the temperature ploted in the Figure 5 inset tells us that at room temperature the PL is independent of the caplayer thickness. At low temperatures, the thicker caplayer gives a stronger PL. We cannot be sure that this trend is maintained for even thicker caplayers.

In a PL experiment, the laser generates electron-hole pairs in the conduction and valence bands of the host semiconductor. In the steady-state PL, all the generated carriers have to recombine through non-radiative and radiative recombination, the latter producing the PL. All these recombination mechanisms (transference of electrons between states) are approximately ruled by diode laws of the difference of the electrochemical potentials (also called quasi-Fermi levels [QFLs]) of the involved states [6,29] (each energy level may be at a different electrochemical potential), usually forward biased (reverse biasing is called generation). The saturation current of the diode laws follows an Arrhenius law, with energy close to the energy difference between the states involved in the transition; this rules, to a large extent, the dependence of the diode on temperature.

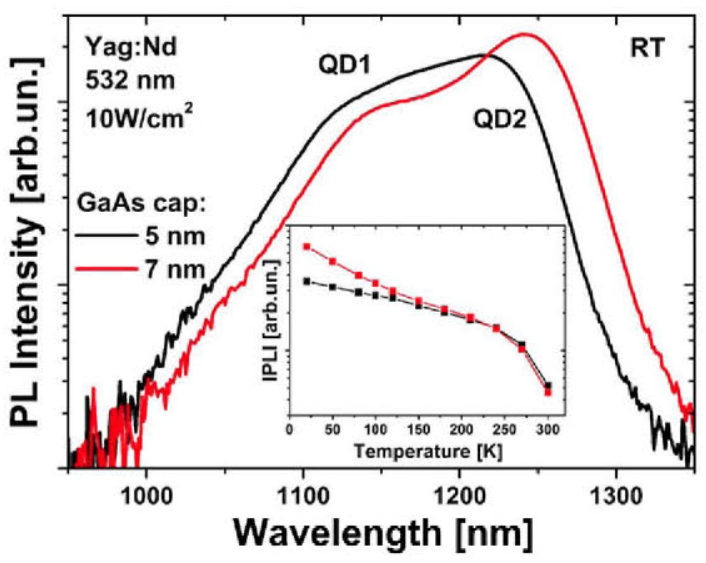

Figure 5. PL spectra of samples with the cap layer thicknesses of 5 and $7 \mathrm{~nm}$ at room temperature. The inset shows the PL integral intensity temperature dependence. 
Because of the Arrhenius-law ruled saturation current, all recombination mechanisms tend to be suppressed (reduced) at low temperature, more suppressed than those with a higher Arrhenius energy, but as the laser produces the same excitation, the splitting of the different pairs of QFLs becomes higher at low temperatures, thus maintaining the same overall recombination rate, although the distribution between transitions is different [29].

There are radiative and non-radiative transitions between the conduction band and the bound states of the QDs, but the latter, producing photons of very low energy, are usually invisible to our PL instruments. They are not important for our discussion here.

At low temperatures, for transitions between the QD bound states and the valence band, the less energetic photons of the QD PL, linking states of less energy difference, are favored because their Arrhenius energy is smaller. At low temperatures, their recombination is less suppressed than the non-radiative recombination, whose Arrhenius energy is usually close to the host bandgap. The lower energy PL recombination increases to conserve the total recombination. That is why at low temperatures, the PL signature of the transition between the QD fundamental states becomes more visible. It is also sharper because the Fermi-Dirac functions governing the population of electrons and holes are sharper.

This is seen in the inset in Figure 5, where the integrated $\mathrm{PL}$ from the QD transitions increases as the temperature is decreased. In the case of the $7 \mathrm{~nm}$ caplayer, the red shift observed in Figure 5 makes the Arrhenius energy smaller, and therefore, the increase in the PL is even larger.

Figure 6(a) presents the PL spectrum of a sample with a caplayer thickness of $7 \mathrm{~nm}$ recorded at different excitation densities and at $20 \mathrm{~K}$. The two QD states, called QD1 and QD2, are clearly visible in all spectra. Peaks of radiation from both the WL and the matrix (GaAs) are also revealed at high excitation densities. The intensity of the peaks possibly reflects the concentration of the QDs of different types. The optical properties of a bimodal QD array are considered in detail in [30].

The evolution of PL spectra with temperatures at a moderate excitation density is presented in Figure 6(b). They can be compared with those produced in classic articles $[31,32]$.

The Arrhenius law - that is, that the transitions with lower Arrhenius energy prevail at low temperatures - applied to explain that the bigger integral PL in the inset of Figure 5 is in reality a rule of thumb. It is not fulfilled in Figure 6(b), where the QD1 peak, presumably of higher Arrhenius energy than the QD2, becomes dominant at low temperature. In this case, the Arrhenius law is refuted, probably through the evolution of the QFLs. A real modeling would require a full detailed balance calculation [21], with data which are seldom available.

In summary, it has been found that highest PL response, which is associated with the best material quality, is obtained by forming QDs on GaAs $6^{\circ}$ substrates, depositing the $\sim 1.7 \mathrm{ML}$ of InAs at a low rate $(0.0625$
$\mathrm{ML} / \mathrm{s}$ ) and capped with a caplayer of $5-7 \mathrm{~nm}$ of $\mathrm{GaAs}$ grown at low temperatures $\left(520^{\circ} \mathrm{C}\right)$ at the rate of $2 \AA / s$. In this case, QDs are probably formed with a multimodal distribution of sizes. A characteristic peculiarity of the QDs grown is also the presence of $\mathrm{Ga}-\mathrm{In}$ intermixing, leading to the formation of a quite voluminous WL.

The conditions determined for the growth of QDs with an intense PL were used for forming multilayer QD arrays (10 and 15 QD layers) and for embedding them into the $\mathrm{GaAs} \mathrm{SC}$ heterostructure. Neither $\mathrm{GaP}$ strain balance layers, like those used in reference [23], nor GaNAs like those in [24] have been used in this case. So, to avoid the strain, wide spacers of $35 \mathrm{~nm}$ have been grown at $700^{\circ} \mathrm{C}$ on the caplayers. The schematic of the structure is presented in Figure 7(a). A TEM of the same structure (for 10 QD layers) is shown in Figure 7(b). No columnar growth in QDs is seen, which proves that the field of strains has faded when the new QD layer starts to grow. Actually, we believe that the columnar growth, often sought, is unnecessary for SCs.

In Figure 8(a), we present a magnification of Figure 7 (b), showing the caplayer and the wetting layer very clearly, together with the InAs QDs. Finally, in Figure 8 (c), we present very high resolution images showing a single QD.
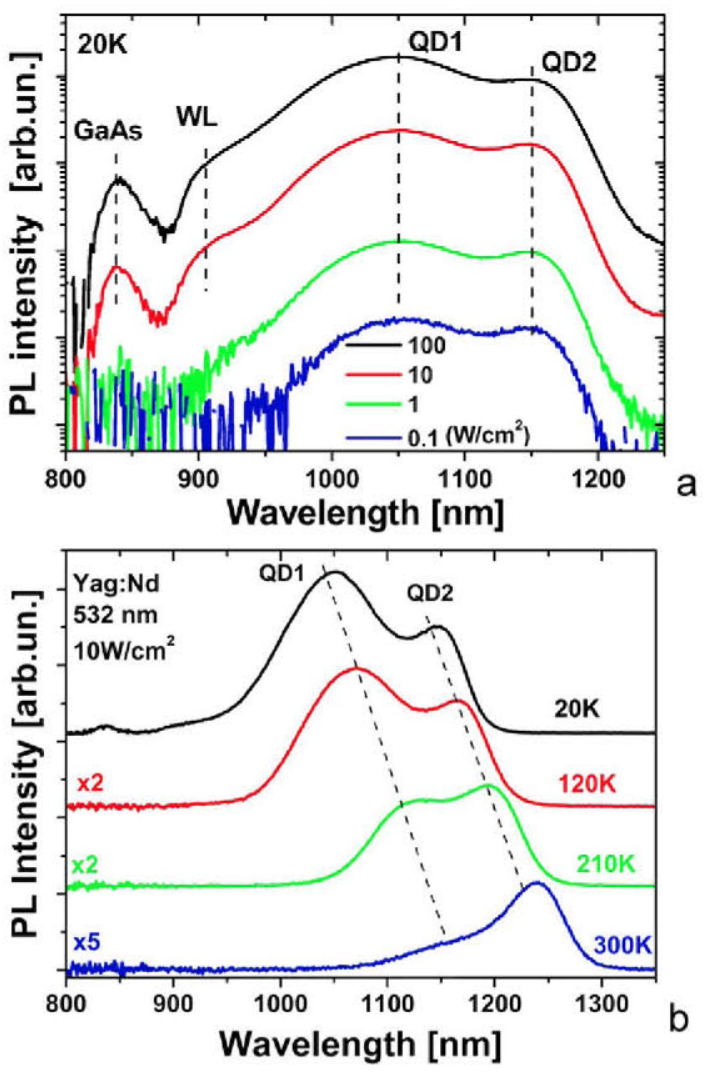

Figure 6. (a) PL spectra for different excitation densities at $20 \mathrm{~K}$; (b) PL spectrum evolution with temperature. The sample cap layer thickness is $7 \mathrm{~nm}$. 


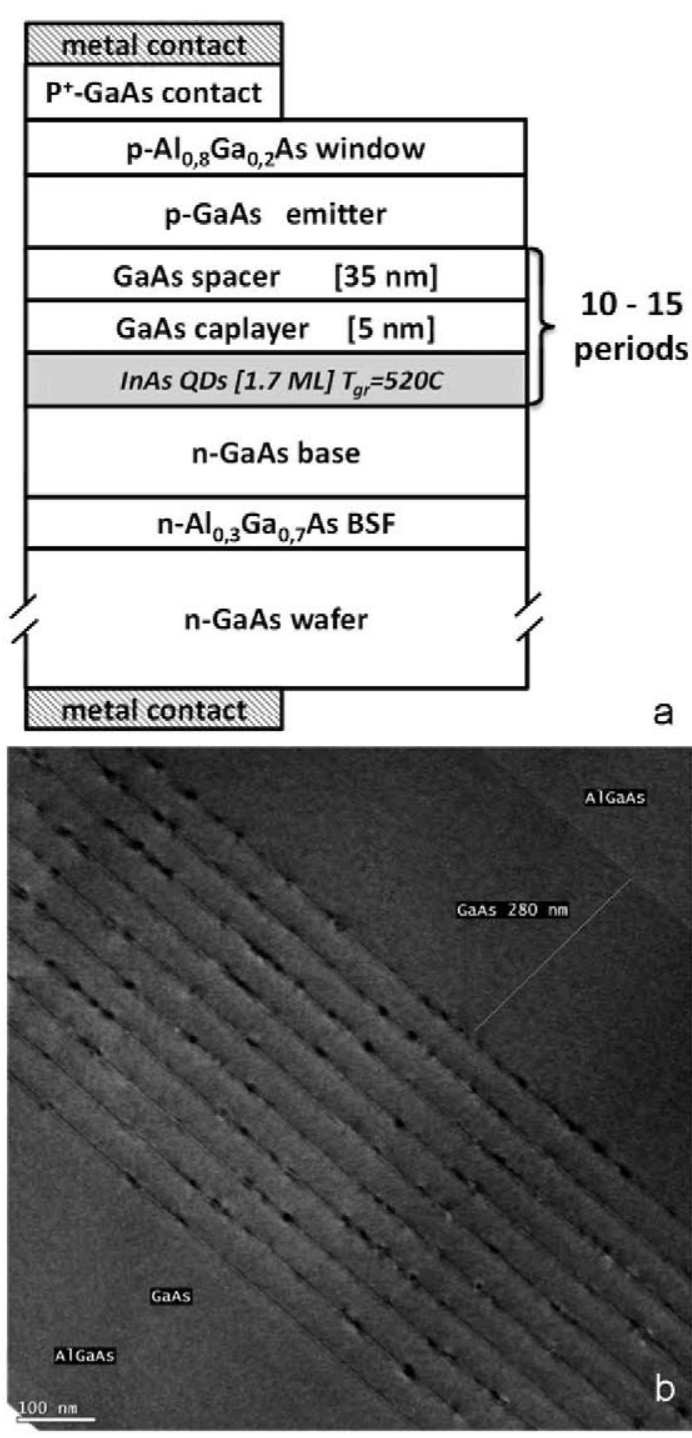

Figure 7. (a) Schematic of solar cell (SC) structure with array of QD layer SC structure and (b) TEM picture, (UZ) showing the grown structure (the direction of growth is from lower left to upper right).

Based on the heterostructures grown, photodiodes with QDs with a configuration of the top metal contact without a grid shadowing the surface have been manufactured. A control photodiode without QDs (reference SC) has also been manufactured. This construction of a photodiode is suitable for spectral response measurements, as there is no shadowing of a surface by contact grid. However, the spreading resistance has a great influence on the voltage characteristics of these photodiodes, even at low concentrations of solar radiation. A preliminary comparison has shown that the open circuit voltage was reduced from $1.02 \mathrm{~V}$ for reference $\mathrm{SC}$ to $0.76 \mathrm{~V}$ for $\mathrm{QD}$ $\mathrm{SC}$ (for a concentration of about 1 sun). This result is correlated, for example, with [22], where a similar decrease in voltage was determined for QD SC with thick spacer

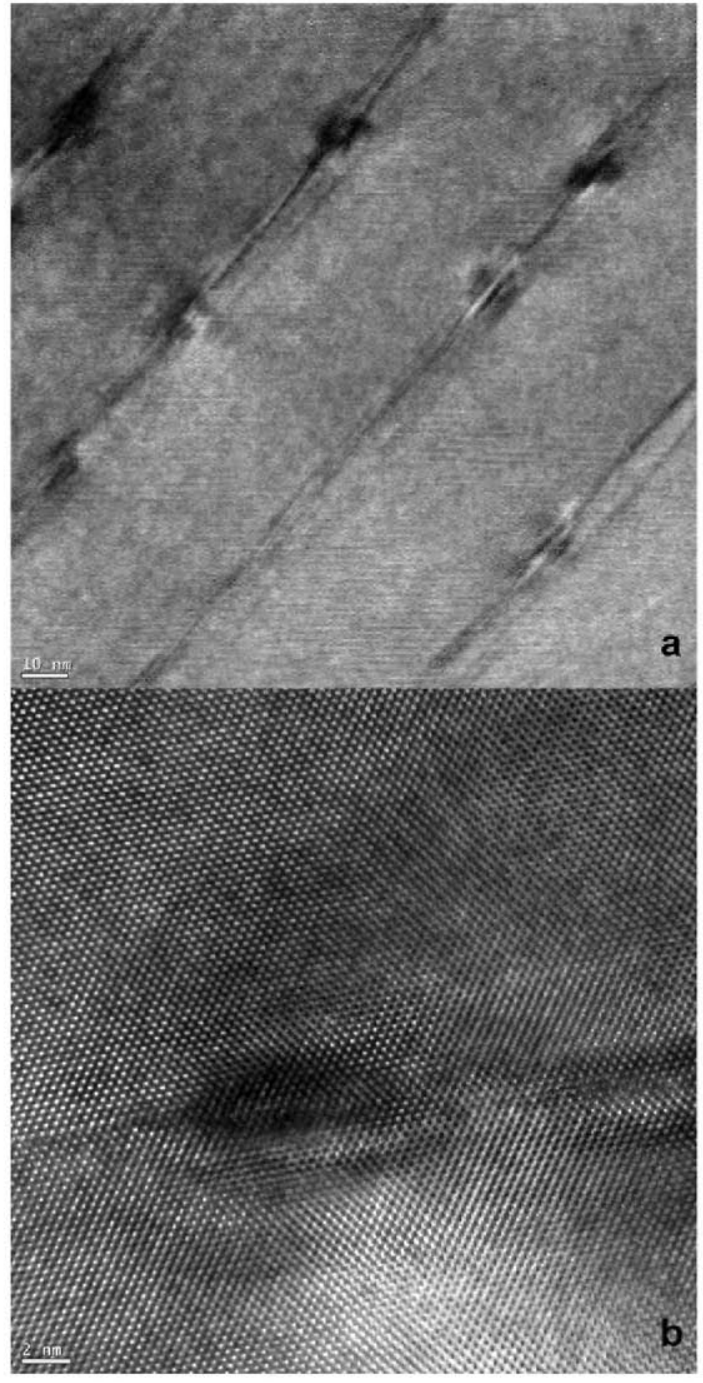

Figure 8. (a) High resolution (UZ) TEM pictures of the sample with the 10xQD array. (b) Detail of one of the QDs.

layers $(40 \mathrm{~nm})$. Spectral dependencies of the EQE and the reflection have been obtained using a control diode. As a result, a spectral dependence of the internal quantum efficiency (IQE) for both samples with QD arrays has been determined (Figure 9).

In the region below $\lambda=880 \mathrm{~nm}$, the spectral dependency of the IQE for a QD SC with 10 QD rows (10xQDs) is at the same level as for the reference sample without QDs. This indicates the high quality of the GaAs p-n junction embedding $10 \mathrm{xQDs}$ layers into the active region even without the application of strain-balance technology. However, for the QD SC with $15 \times$ QDs, a drop in the IQE in the spectral range below $\lambda=880 \mathrm{~nm}$ is observed. This leads to a decrease in the value of the photocurrent $\left(J_{\mathrm{g}}\right)$ for this sample compared with the $10 \mathrm{xQDs} \mathrm{SC}$ despite the increase in $J_{\mathrm{g}}$ in the spectral range of $880-1080 \mathrm{~nm}$ (Figure 10). However, this might be a contingent situation, and many SC samples should undergo 


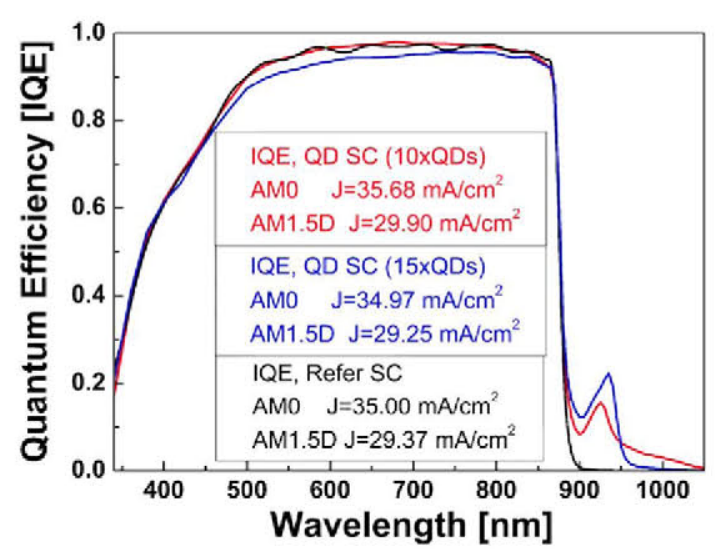

Figure 9. Spectral dependences of internal quantum efficiency for QD SCs with 10-layer and 15-layer QD arrays and for a reference SC without $\mathrm{QD}$. Photogenerated currents $(J)$ are presented in the boxes.

statistical tests to determine the best number of layers in the stack.

On the whole, the photocurrent values obtained by integrating the spectral dependence with the IQE in the range of $880-1200 \mathrm{~nm}$ for the heterostructure of a QD SC with $10 x Q D s c$ are about $0.577 \mathrm{~mA} / \mathrm{cm}^{2}$ for the AM1.D spectrum and about $0.744 \mathrm{~mA} / \mathrm{cm}^{2}$ for that of the AM0. This corresponds to about $0.06 \mathrm{~mA} / \mathrm{cm}^{2}$ and $0.07 \mathrm{~mA} / \mathrm{cm}^{2}$ per a QD layer for AM1.D and AM0 spectra, respectively.

For the $15 \mathrm{xQD} \mathrm{SC}, J_{\mathrm{g}}(\lambda=880-1200 \mathrm{~nm})$ is $0.592 \mathrm{~mA} /$ $\mathrm{cm}^{2}$ for the AM1.D spectrum and $0.815 \mathrm{~mA} / \mathrm{cm}^{2}$ for that of the AM0. This corresponds to about $0.04 \mathrm{~mA} / \mathrm{cm}^{2}$ per a QD layer and about $0.05 \mathrm{~mA} / \mathrm{cm}^{2}$ per a QD layer for the AM1.D and AM0 spectra, respectively.

To our knowledge, these photocurrent values for the QD arrays with 10-15xQDs per layer are the highest achieved so far for InAs QDs in a barrier material of GaAs.

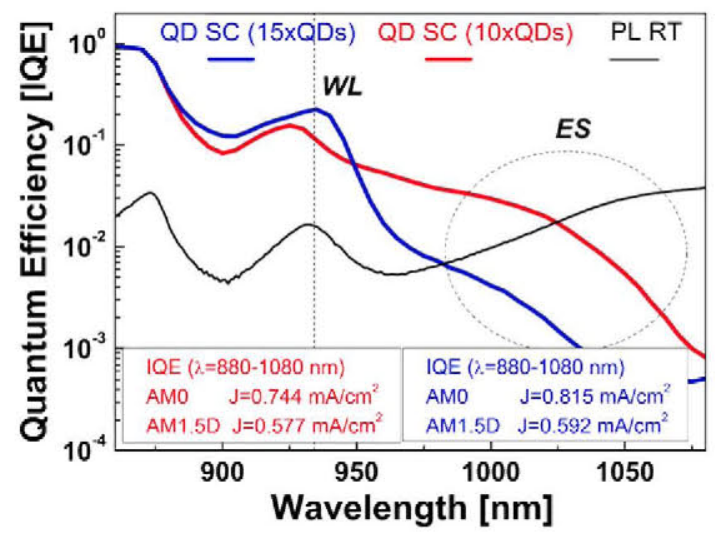

Figure 10. Spectral dependences of internal quantum efficiency of QD SCs with 10xQDs and 15xQDs and for a reference SC without QDs. We also present the room temperature PL of the QD multilayer structure. The wetting layer is clearly visible as well as the first excited state of the QDs (inside the ES circle).
Two peaks in the IQE spectral dependencies for both QD SCs beyond the GaAs absorption edge are observed in the range of $880-1080 \mathrm{~nm}$. A comparison with the PL spectrum shows that according to the wavelength, the first peak is determined by the WL. The second QE peak, apparently, is associated with the QD1 peak, of lower energy, associated, as we have said, to the smaller QDs. Data on the behavior of the QD2, which would be the largest at room temperature, are unfortunately beyond the range of our measurements.

With the caution of working on a single sample, it is also seen that by raising the number of QD layers, strain in the structure increases, which leads not only to reducing the IQE in the spectral region below $880 \mathrm{~nm}$ (Figure 9) but also decreasing the contribution of states in the QDs themselves (the blue line in Figure 10). The main increase in the QD SC (15xQDs) structure photocurrent is achieved at the cost of increasing the number of WLs. Thus, further increasing the number of QD layers in an array should probably be carried out using strain-balance layers.

In summary, we think that the developed MOVPE QD technology is promising for manufacturing lattice matched multi-junction SCs with improved photocurrent matching as a result of the increase in $J_{\mathrm{g}}$ in the middle subcell similar to the QD SC prototypes manufactured here.

\section{CONCLUSION}

The conditions for manufacturing QD SCs with the purpose of producing IB SCs, as well as extending the spectral response of GaAs SCs to be included in MJ stacks, have been researched. The growth rate of the GaAs matrix material has been keep high, above $1.5 \mu \mathrm{m} / \mathrm{h}$, as a technological constraint for cost considerations.

In the manufacturing of the InAs QD structure, the highest PL response is identified with the lowest non-radiative recombination material. It has been found that the highest PL response is obtained by forming QDs on GaAs $6^{\circ}$ substrates, depositing the $\sim 1.7 \mathrm{ML}$ of InAs at low rate $(0.0625 \mathrm{ML} / \mathrm{s})$ and covered with a caplayer of $5-7 \mathrm{~nm}$ of GaAs grown al low temperature $\left(520^{\circ} \mathrm{C}\right)$ at the rate of $2 \AA / \mathrm{s}$. In this case, the QDs are presumably formed with a multimodal distribution of sizes.

Solar cell structures with 10 and 15 layers of QDs have been grown using the aforementioned methodology, together with a control cell without QDs. The QD cells show an excellent IQE with no degradation with respect to the control cell for those of 10 QD layers; some degradation is observed in the 15 QD layer cells. In addition, they present an excellent sub-bandgap current photo-response amounting to $0.07 \mathrm{~mA} / \mathrm{cm}^{2}$ per a QD layer, to our knowledge, the highest value so far achieved in InAs QDs embedded in pure GaAs.

We consider that the procedure is attractive in improving the efficiency of the lattice matched multi-junction SCs and is also helpful in the achievement of high-quality QDs in III-V semiconductors, useful for IB SCs. 


\section{ACKNOWLEDGEMENTS}

The authors wish to thank G.V. Il'menkov for valuable discussions, N. Kh. Timoshina for the spectral dependence measurements, and to Alfonso Ibarra for the TEM pictures of the University of Zaragoza.

The testing of heterostructures using the TEM and AFM methods was carried out on the equipment of the Joint Research Centre «Material Science and Characterization in Advanced Technology» (Ioffe Institute). The Polytechnic University of Madrid provided the full stack TEM images.

The authors want to acknowledge that this work has been supported by the contract no. 14.B25.31.0020 from the Russian Ministry of Education and Science (resolution no. 220) and contract no. ENE2012-37804-C02-01 from the Spanish National Research Plan.

\section{REFERENCES}

1. Green MA. Third generation photovoltaics: solar cells for 2020 and beyond. Physica E: Low-dimensional Systems and Nanostructures 2002; 14(1-2): 65-70.

2. Luque A, Martí A. Increasing the efficiency of ideal solar cells by photon induced transitions at intermediate levels. Physical Review Letters 1997; 78: 5014 .

3. Nozik AJ. Quantum dot solar cells. Physica 2002; E 14(1-2): 115-120.

4. Marti A, Cuadra L, Luque A. IEEE: quantum dot intermediate band solar cell. in: Conference Record of the Twenty-Eighth IEEE Photovoltaic Specialists Conference 2000. IEEE, New York (2000), 940-943.

5. Luque A, Marti A, Nozik AJ. Solar cells based on quantum dots: multiple exciton generation and intermediate bands. MRS Bulletin 2007; 32(3): 236-241.

6. Luque A, Marti A, Stanley C. Understanding intermediate-band solar cells. Nature Photonics 2012; 6: 146 .

7. Semonin OE, Luther JM, Choi S, Chen HY, Gao JB, Nozik AJ, Beard MC. Peak external photocurrent quantum efficiency exceeding $100 \%$ via $\mathrm{MEG}$ in a quantum dot solar cell. Science 2011; 334(6062): 1530-1533. DOI:10.1126/science. 1209845.

8. Luque A, Martí A, Stanley C, López N, Cuadra L, Zhou D, Pearson JL, McKee A. General equivalent circuit for intermediate band devices: potentials, currents and electroluminescence. Journal of Applied Physics 2004; 96: 903 .

9. Ramiro I, Martí A, Antolín E, Luque A. Review of experimental results related to the operation of intermediate band solar cells. IEEE Journal of Photovoltaics $2014 ;$ 4(2): 736-748.
10. Blokhin SA, Sakharov AV, Nadtochy AM, Pauysov AS, Maximov MV, Ledentsov NN, Kovsh AR, Mikhrin SS, Lantratov VM, Mintairov SA, Kaluzhniy NA, Shvarts MZ. AlGaAs/GaAs. Photovoltaic cells with an array of InGaAs QDs. Semiconductors 2009; 43: 514.

11. Ramiro I, Antolin E, Steer MJ, Linares PG, Hernandez E, Artacho I, Lopez E, Ben T, Ripalda JM, Molina SI, Briones F, Stanley CR, Marti A, Luque A. InAs/ AlGaAs quantum dot intermediate band solar cells with enlarged sub-bandgaps. 38th IEEE Photovoltaic Specialists Conference. New York: IEEE, 2012, 652.

12. Ramiro I, Villa J, Lam P, Hatch S, Wu J, Lopez E, Antolin E, Liu H, Marti A, Luque A. Wide-bandgap InAs/InGaP quantum-dot intermediate band solar cells. IEEE Journal of Photovoltaics 2015; 5(3): 840-845.

13. Ledentsov NN, Ustinov VM, Shchukin VA, Kop'ev PS, Alferov ZI, Bimberg D. Quantum dot heterostructures: fabrication, properties, lasers (review). Semiconductors 1998; 32(4): 343.

14. Mikhrin SS, Zhukov AE, Kovsh AR, Maleev NA, Vasil'ev AP, Semenova ES, Ustinov VM, Kulagina MM, Nikitina EV, Soshnikov IP, Shernyakov YM, Livshits DA, Kryjanovskaya NV, Sizov DS, Maksimov MV, Tsatsul'nikov AF, Ledentsov NN, Bimberg D, Alferov ZI. High efficiency (eta(D)> $80 \%$ ) long wavelength (lambda $>1.25 \mathrm{mu} \mathrm{m}$ ) quantum dot diode lasers on GaAs substrates. Semiconductors 2002; 36(11): 1315.

15. Mikhrin SS, Kovsh AR, Krestnikov IL, Kozhukhov AV, Livshits DA, Ledentsov NN, Shernyakov YM, Novikov II, Maximov MV, Ustinov VM, Alferov ZI. High power temperature-insensitive $\quad 1.3 \mu \mathrm{m} \quad \mathrm{InAs} / \mathrm{InGaAs} / \mathrm{GaAs}$ quantum dot lasers. Semiconductor Science and Technology 2005; 20: 340 .

16. Kryzhanovskaya NV, Mukhin IS, Moiseev EI, Shostak II, Bogdanov AA, Nadtochiy AM, Maximov MV, Zhukov AE, Kulagina MM, Vashanova KA, Zadiranov YM, Troshkov SI, Lipovskii AA, Mintairov A. Control of emission spectra in quantum dot microdisk/microring lasers. Optics Express 2014; 22(21): 25782.

17. Kovsh A, Krestnikov I, Livshits D, Mikhrin S, Weimer J, Zhukov A. Quantum dot laser with $75 \mathrm{~nm}$ broad spectrum of emission. Optics Letters 2007; 32: 793.

18. Patel P, Aiken D, Boca A, Cho B, Chumney D, Clevenger B, Cornfeld A, Fatemi N, Lin Y, McCarty J, Newman F, Sharps P, Spann J, Stan M, Steinfeldt J, Varghese T. Experimental results from performance improvement and radiation hardening of inverted metamorphic multi-junction solar cells. Presented at the Photovoltaic Spec. Conf., Seattle, WA 2011.

19. Cornfeld AB, Stan M, Varghese T, Diaz J, Ley AV, Cho B, Korostyshevsky A, Aiken DJ, Sharps PR. 
Development of a large area inverted metamorphic multi-junction (IMM) highly efficient AM0 solar cell, Photovoltaic Specialists Conference, PVSC '08. 33rd IEEE 2008; 26, DOI: 10.1109/PVSC.2008.4922610

20. Hubbard SM, Cress CD, Bailey CG, Raffaelle RP, Bailey SG, Wilt DM. Effect of strain compensation on quantum dot enhanced GaAs solar cells. Applied Physics Letters 2008; 92(12): 123512.

21. Guimard D, Morihara R, Bordel D, Tanabe K, Wakayama Y, Nishioka M, Arakawa Y. Fabrication of InAs/GaAs quantum dot solar cells with enhanced photocurrent and without degradation of open circuit voltage. Applied Physics Letters 2010; 96: 203507.

22. Tanabe K, Guimard D, Bordel D, Arakawa Y. Highefficiency InAs/GaAs quantum dot solar cells by metalorganic chemical vapor deposition. Applied Physics Letters 2012; 100: 193905.

23. Bailey CG, Forbes DV, Polly SJ, Bittner ZH, Dai Y, Mackos C, Raffaelle RP, Hubbard SM. Open-circuit voltage improvement of InAs/GaAs quantum-dot solar cells using reduced InAs coverage. IEEE Journal of Photovoltaics 2012; 2(3): 269-275.

24. Oshima R, Takata A, Okada Y. Strain-compensated InAs/GaNAs quantum dots for use in high-efficiency solar cells. Applied Physics Letters 2008; 93: 083111.

25. Salii RA, Mintairov SA, Brunkov PN, Nadtochiy AM, Payusov AS, Kalyuzhnyy NA. Determination of growth parameters in the InAs-GaAs system for the formation of InAs quantum dots with multi-modal distribution by MOCVD technique. Semiconductors 2015; 49(8): 1136-1143.
26. Stringfellow GB. Organometallic Vapor-Phase Epitaxy: Theory and Practice, 2nd edn. Academic Press: San Diego, 1999. 370.

27. Sears K, Mokkapati S, Tan HH, Jagadish C. In (Ga)As/GaAs quantum dots grown by MOCVD for opto-electronic device applications Chapter 12, in the Book. In Self-Assembled Quantum Dots, Wang ZM (eds). Springer: New York, USA, 2008; $359-403$.

28. Goldstein L, Glas F, Marzin JY, Charasse MN, Le Roux G. Growth by molecular beam epitaxy and characterization of InAs/GaAs strained-layer superlattices. Applied Physics Letters 1985; 47(10): 1099.

29. Mellor A, Luque A, Tobías I, Martí A. Realistic detailed balance study of the quantum efficiency of quantum dot solar cells. Advanced Functional Materials 2014; 24: 339.

30. Bansal B. A model for the temperature dependence of photoluminescence from self-assembled quantum dots. Journal of Applied Physics 2006; 100: 093107.

31. Fafard S, Wasilewski ZR, Allen CN, Picard D, Spanner M, McCaffrey JP, Piva PG. Manipulating the energy levels of semiconductor quantum dots. Physical Review B 1999; 59: 15368-15373.

32. Kryzhanovskaya NV, Gladyshev AG, Blokhin SA, Maksimov MV, Semenova ES, Vasil'ev AP, Zhukov AE, Ledentsov NN, Ustinov VM, Bimberg D. Nonequilibrium room-temperature carrier distribution in InAs quantum dots overgrown with thin AlAs/InAlAs layer. Semiconductors 2005; 39: 1188-1193. 